\title{
Magnetic field dependence of the energy of negatively charged excitons in semiconductor quantum wells.
}

\author{
C. Rivat and F. M. Peeters \\ Departement Natuurkunde, Universiteit Antwerpen (UIA), Universiteitsplein 1, B-2610 Antwerpen, Belgium. \\ K. Vargal \\ Solid State Division, Oak Ridge National Laboratory, Oak Ridge, Tennessee 37831-3062
}

(October 29, 2018)

\begin{abstract}
A variational calculation of the spin-singlet and spin-triplet state of a negatively charged exciton (trion) confined to a single quantum well and in the presence of a perpendicular magnetic field is presented. We calculated the probability density and the pair correlation function of the singlet and triplet trion states. The dependence of the energy levels and of the binding energy on the well width and on the magnetic field strength was investigated. We compared our results with the available experimental data on GaAs/AlGaAs quantum wells and find that in the low magnetic field region $(B<18 \mathrm{~T})$ the observed transition are those of the singlet and the dark triplet trion (with angular momentum $\left.L_{z}=-1\right)$, while for high magnetic fields $(B>25 \mathrm{~T})$ the dark trion becomes optically inactive and possibly a transition to a bright triplet trion (angular momentum $L_{z}=0$ ) state is observed.

PACS number: $71.35,78.66 . \mathrm{Fd}, 78.55$
\end{abstract}

\section{INTRODUCTION}

After the initial work by Lampert, 1 who proved the stability of the charged exciton fomplexes, charged excitons in bulk semiconductors These studies revealed that, due to the confinement, the 2D charged excitons have binding energies which are an order of magnitude larger than charged excitons in the corresponding bulk materials. The increased binding energy in reduced dimensionality systems together with the improved experimental techniques have allowed the experimentalists to observe them in quantum well structures $\mathbf{0}$ Many of the experimental results reported in the literature are for charged excitons in the presence of a perpendicular magnetic field 611 Up to recently, there was little or no agreement between the experimental results and the available theories.12.13

Lately, however, progress was made in the direction of bringing theoretical prediction and experiments closer to each other. Stébé and Moradi13 used a variational method which was valid in the low magnetic field regime and explained the minimum around 1 Tesla observed experimentally by Shields et al.11 in the charged exciton singlet transition energy for a $300 \AA$ wide quantum well. Recently Muntenau et al 14 found a transition between the singlet ground state and the triplet ground state at $B=35 \mathrm{~T}$ for a $200 \AA$ wide asymmetric quantum well, similar to the one predicted earlier by Whittaker and Shields 12 for a $100 \AA$ wide symmetric quantum well.

The triplet transition energies which have been so far identified are assigned to the anoular momentum $L_{z}=-1$ triplet state. In exactly 2D systems with translational invariance this state was shown 15.16 to be an optically dark state. As a consequence, one would expect that such a state is 'dark' also in quasi-2D systems, particularly in narrow quantum wells. The fact that the $L_{z}=-1$ triplet is observed in quantum wells suggests that a breaking of symmetry occurs and in particular that the system is no longer invariant under a magnetic translation. Recently, the existence of a bound bright triplet state, i.e. $L_{z}=0$ was predicted.17 Due to its small binding energy, this triplet state could be difficult to detect. The possible existence of such a triplet state may force us to review the assignment that has been made of some of the photoluminescence lines.

Our previous works $\mathbf{t}, \mathrm{Lg}$ on charged excitons in quantum wells was limited to the case of zero magnetic field and showed that the stochastic variational method (SVM) is an efficient technique for solving the effective mass Hamiltonian of exciten complexes without involving any approximations. In Ref. 19 we showed that approximations made by Stébé et al.20 in the Coulomb matrix elements lead to an overestimation of the trion binding energy. The latter approximation aimed to convert the problem into an effective 2D problem. In our approach no simplifying approximations are made and the full 3D nature of the quantum well problem is retained. Here we extend our previous work to the important experimental situation in which an uniform magnetic field is applied along the quantum well growth axis. Our results for the magnetic field dependence of the trion singlet binding energy agrees, for the first time, with available experimental results on $100 \AA$ and $300 \AA$ wide GaAs/AlGaAs quantum wells. Furthermore we find that the earlier predicted bright triplet is unbound for the $300 \AA$ wide quantum well and probably marginally bound for the $100 \AA$ wide quantum well. 
The present paper is organized as follows. In Sec. II we present the Hamiltonian of the problem and outline our method to obtain the energy of the exciton and charged exciton. The conditional probability density function of the trion, its pairs correlation functions and the average distance between the different particles in the trion are discussed in Sec. III. In Sec. IV we compare our results for the transition energy and in Sec. V for the binding energy with available experimental data on symmetric GaAs/AlGaAs quantum wells and with the theoretical results of Whittaker and Shields.12 In the last section we summarize our results and present our conclusions.

\section{THE MODEL}

In the effective mass approximation the Hamiltonian describing a negative charged exciton, i.e. $\mathrm{X}^{-}$, in an uniform magnetic field $\mathrm{B}$ is given by

$$
H=\sum_{i=1}^{3} \frac{1}{2 m_{i}}\left(\vec{p}_{i}-\frac{e_{i}}{c} \vec{A}_{i}\right)^{2}+\sum_{i=1}^{3} V\left(\vec{r}_{i}\right)+\sum_{i<j} \frac{e_{i} e_{j}}{\varepsilon\left|\vec{r}_{i}-\vec{r}_{j}\right|},
$$

where $\vec{A}_{i}=\frac{1}{2} \vec{r}_{i} \times \vec{B}$ is the vector potential; $m_{i}, e_{i}$ are the masses and charges of the interacting particles; $\varepsilon$ is the dielectric constant; the confinement potential is $V\left(\mathbf{r}_{i}\right)=0$ if $|z|<W / 2$ and $V\left(\mathbf{r}_{i}\right)=V_{i}$ if $|z|<W / 2$, with $W$ the quantum well width. The reference system is taken such that the origin of the coordinate system is at the center of the quantum well. For a $\mathrm{GaAs} / \mathrm{Al}_{x} \mathrm{Ga}_{1-x}$ As quantum well the heights of the square well confinement potentials are $V_{e}=0.57 \times\left(1.155 x+0.37 x^{2}\right) \mathrm{eV}$ for the electrons and $V_{h}=0.43 \times\left(1.155 x+0.37 x^{2}\right) \mathrm{eV}$ for the hole. If we consider the case where the magnetic field is applied along the growth axis of the well, i.e. $\vec{B}=(0,0, B)$, the Hamiltonian becomes:

$$
H=\sum_{i=1}^{3} \frac{1}{2 m_{i}}\left(-\hbar^{2} \Delta_{i}+\frac{e_{i}^{2} B^{2}}{4 c^{2}}\left(x_{i}^{2}+y_{i}^{2}\right)-\frac{e_{i} \hbar B}{c} l_{z i}\right)+\sum_{i=1}^{3} V\left(\vec{r}_{i}\right)+\sum_{i<j} \frac{e_{i} e_{j}}{\left|\vec{r}_{i}-\vec{r}_{j}\right|},
$$

where $l_{z i}=-i \partial / \partial \phi_{z i}$ is the $z$-component of the orbital momentum of the $i$-th particle. The Hamiltonian under examination has cylindrical symmetry with respect to the quantum well axis, i.e. $z$-axis, which implies that the z-component of the total orbital angular momentum, $L_{z}$, is a conserved quantity, i.e. a good quantum number. The spin interaction is not explicitly included in our Hamiltonian. The total spin of the electrons, $S_{e}$, and the spin of the hole, $S_{h}$, and their projections along the z-axis, $S_{h z}$ and $S_{e z}$, are conserved quantities. Notice that the state of the system is not degenerate with respect to the total electron spin. In fact the two electrons obey Fermi-Dirac statistics which means that the electronic part of the total wave function must be antisymmetric, i.e. when $S_{e}=0$ the spatial part of the electronic wave function must be symmetric and when $S_{e}=1$ the spatial part of the electronic wave function must be anti-symmetric. Thus, $S_{e}$ can be used as a quantum number which indicates the parity of the state. Once the projection along $z$ of the total orbital momentum, $L_{z}$, and the electron spin $S_{e}$ are fixed we obtain, after solving our Hamiltonian, a series of energy levels which we indicate by the quantum numbers $\left(n, L_{z}, S_{e}\right)$, where $n$ is the principal quantum number. These levels are degenerate with respect to the quantum number $S_{h}, S_{h z}$ and $S_{e z}$.

The Hamiltonian (2) is solved using the stochastic variational method which was outlined in Ref. 21. The trial function, for the variational calculation, is taken as a linear combination of "deformed" correlated Gaussian functions (DCG),

$$
\phi_{N}\left(\vec{r}_{1 e}, \vec{r}_{2 e}, \vec{r}_{h}\right)=\sum_{m=1}^{K} C_{q N} \Phi_{q N}\left(\vec{r}_{1 e}, \vec{r}_{2 e}, \vec{r}_{h}\right)
$$

with

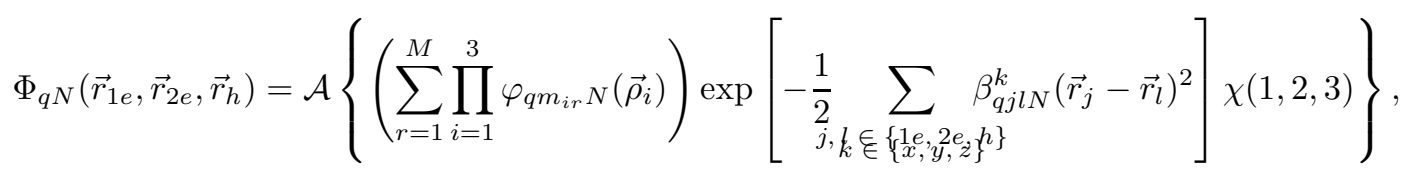

and

$$
\varphi_{q m_{i r} N}\left(\vec{\rho}_{i}\right)=\xi_{q m_{i r}}\left(\vec{\rho}_{i}\right) \exp \left(-\sum_{k \in\{x, y, z\}} \beta_{q i i N}^{k} r_{i i}^{2}\right)
$$


where $r_{i k}$ gives the position of the $i$-th particle in the $k$-direction; $\mathcal{A}$ is the antisymmetrization operator and $\left\{C_{q N}, \beta_{q i l N}^{k}\right\}$ are the variational parameters, $\chi(1,2,3)$ is the three particle spin function, and $\xi_{q m_{i r}}(\vec{\rho})=(x+i y)^{m_{i r}}$ with $m_{i r}$ integers such that $L_{z}=m_{1 r}+m_{2 r}+m_{3 r}$ for each value of $r$, with $L_{z}$ the projection of the total angular momentum along the $z$-axis; $M$ is the number of channels used to obtain our state; $N$ indicates for brevity the set of quantum numbers which characterizes our state, i.e. $\left(n, L_{z}, S_{e}\right)$. Note that in contrast to the "classical" correlated Gaussians, here, the parameter $\beta_{q j l N}^{k}$ which expresses the correlation among the particle $j$ and the particle $l$ in the direction $k$, is allowed to be different from the parameter $\beta_{q j l N}^{k^{\prime}}$ which couples the same two particles $j$ and $l$ in a different direction $k^{\prime}$. This additional degree of freedom in the calculation allows us to take into account the asymmetry introduced in the 3D space by the presence of the quantum well and of the magnetic field.

A basis of dimension $K$, e.g. 10, is at first selected using the stochastic procedure. This does not ensure that the best basis set is found, so a refinement procedure is carried out on the basis set in order to improve it. The refinement is made by replacing the $m$-th state with a new state, i.e. with a state built using new parameters $\left\{C_{m N}, \beta_{m i l N}^{k}\right\}$ in such a way that the total energy is lowered. When the refinement process does not change the total energy significantly, the number of basis states is further increased. The process is reiterated multiple times for different and increasingly larger dimensions of the basis set, until the energy reaches the desired accuracy. The final dimension of the basis set consists typically of 400 states. Faster convergence is obtained by taking into account the cylindrical symmetry, i.e. by choosing $\beta_{q j l N}^{x}=\beta_{q j l N}^{y}$. Notice also that with respect to the case without magnetic field, less basis states have to be used because the magnetic field localizes the particles around the magnetic center of mass leading to a faster convergence of the energy. The number of channels used depends on the magnetic field. For example, for the case $L_{z}=0$, we found that for low magnetic fields we already obtain good results using one channel, which actually gives the largest contribution, while for large fields we have to use up to 7 channels, to obtain a reasonable convergence. On the other hand, for small magnetic fields we need larger number of states $K$ in order to accurately describe the trion energy.

\section{THEORETICAL RESULTS}

Our numerical results are given for a $\mathrm{GaAs} / \mathrm{Al}_{x} \mathrm{Ga}_{1-x} \mathrm{As}$ quantum well. The parameter used in our calculation are $x=0.3, \varepsilon=12.58$ and $m_{e}=0.067 m_{0}$, which give for our unit of length $a_{B}=\epsilon \hbar^{2} / e^{2} m_{e}=99.3 \AA$ and energy $2 R_{y}=e^{2} / \epsilon a_{B}=11.58 \mathrm{meV}$. Notice that $R_{y}$ and $a_{B}$ are calculated for the donor problem and do not depend on the hole mass which we took to be $m_{h}=0.34 m_{0}$. Often one uses $a_{B}^{*}=\epsilon \hbar^{2} / e^{2} \mu_{e}$ and $R_{y}^{*}=e^{2} / \epsilon a_{B}^{*}$ where $\mu$ is the exciton reduced mass, i.e. $1 / \mu=1 / m_{e}+1 / m_{h}$, which for our problem is $\mu=0.056 m_{0}$ corresponding to $a_{B}^{*}=118 \AA$ and $R_{y}^{*}=4.8 \mathrm{meV}$.

First we studied the magnetic field dependence of the interparticle average distance. In Fig. 目 we present the 2D average distance, $d_{i j}=\left\langle{\overrightarrow{\rho_{i j}}}^{2}\right\rangle^{1 / 2}$, vs. the magnetic field for the electron-electron pair and for the electron-hole pair, both in the $\left(n=0, L_{z}=0, S_{e}=0\right)$ state, i.e. the singlet (solid curves) and in the $\left(n=0, L_{z}=-1, S_{e}=1\right)$ state, i.e. the triplet (dashed curves) for a $100 \AA$ wide quantum well. As a comparison we show also the exciton electron-hole interparticle distance vs. magnetic field. For the exciton problem the electron and the hole are more strongly bound and the interparticle distance decreases more slowly than for the trion's singlet and triplet state. Nevertheless, it decreases by $50 \%$ over the magnetic field range shown in the figure. For the negatively-charged exciton the electron-electron average distance is always larger than the electron-hole average distance both for the electron spin-singlet state and for the electron spin-triplet state. This of course is a consequence of the repulsive electron-electron interaction, while the electron-hole is attractive. Notice that for $B=0$ the electron-hole distance for the negative charged exciton is about twice the exciton one. The triplet state is more than 20 times larger than the singlet-state in the small magnetic field range where the triplet state is, in fact, unbound. The size of the charged exciton decreases with increasing magnetic field. This decrease is faster in the low magnetic field region, and it is faster for the triplet than for the singlet state. The reason is that the triplet state is more extended, it is less bound, and consequently an external magnetic field will have a larger effect on its size. Notice also that for both states, i.e. singlet and triplet, the curves for $d_{e e}$ and $d_{e h}$ are almost parallel to each other, but nevertheless with increasing magnetic field the distance between them slowly decreases.

Next we calculated the 2D pair correlation function, $g_{i j}^{2 D}(\rho)=\left\langle\delta\left(\left|\vec{\rho}_{i}-\vec{\rho}_{j}\right|-\rho\right)>\right.$, for the spin-singlet and spin-triplet state of a charged exciton in a quantum well of width $100 \AA$ in a magnetic field of $\mathrm{B}=13.7 \mathrm{~T}$, see Fig. 2. We notice that the electron-hole pair correlation function both for the spin-singlet state (dashed curve) and for the spin-triplet state (dash-dotted curve) has its maximum when the distance between the particles is zero. This means that in both states the electron and hole have the tendency of staying close to each other. Notice that the triplet electron-hole pair has a longer tail compared to the singlet one, indicating that the triplet is more extended but, nevertheless, the particles in 
this state are still correlated even at large distances. On the other hand the electron-electron pair correlation function in the singlet state (solid curve) shows that, even though the electrons have a significant probability of being close to each other, the correlation is maximal for $\rho=0.35 a_{B}$ which is a consequence of the Coulomb repulsion between the electrons. In the triplet state the pair correlation function is zero if the particles are in the same position in space, which is an expression of the Pauli exclusion principle, and has a maximum at $\rho=1.32 a_{B}$.

To gain further understanding on how the system is influenced by the presence of a magnetic field, we studied the conditional probability, which gives the probability of finding one of the three particles in position $\vec{r}$ when the other two particles are fixed at $\vec{r}_{1,0}$ and $\vec{r}_{2,0}$. Notice that by fixing two of the particles we obtain information on the positional correlation of the third particle. We focus on the $\hat{x y}$-correlation since the effect of the applied magnetic field along the quantum well axis is larger in the plane orthogonal to the quantum well axis. Along the $z$-direction the probability is mainly determined by the confinement potential. Because the $x$ - and $y$-axis are equivalent due to the cylindrical symmetry of the problem we take $\vec{r}=(x, 0,0)$ for all three particles and for brevity we will indicate $\left|\Phi\left(\vec{r}, \vec{r}_{1,0}, \vec{r}_{2,0}\right)\right|^{2}$ by $|\Phi(x, 0,0)|^{2}$. In Fig. $\beta(\mathrm{a}, \mathrm{b}, \mathrm{c})$ we plot $|\Phi(x, 0,0)|^{2}$ for a negatively charged exciton in a $100 \AA$ wide quantum well when the two electrons are fixed at a distance given by their average distance $d_{e e}=<\rho_{e e}^{2}>^{1 / 2}$. Notice that for $B=0 \mathrm{~T}$ (Fig. 3(a)) the hole is centered around each of the two electrons, while for $B=13.7 \mathrm{~T}$ and for $B=54 \mathrm{~T}$ (Fig. B (b,c)) the hole is mostly situated in the region between the two electrons. For $B=0 \mathrm{~T}$ there is a smaller but not zero probability that the hole is between the two electrons. This binds the two electrons together. When a magnetic field is applied the electrons are on the average closer to each other and as a consequence the two "hole clouds" around the electrons overlap. The hole has almost the same probability of sitting on top of the two electrons or between them. Notice, that when a magnetic field is applied the conditional probability still shows two "kinks" at the position of the two electrons, which are memories of the two peaks present in the conditional probability function at $B=0$. Furthermore, for increasing $B$ the hole wave function decays much faster when the hole moves away from the electron. The increased probability for the hole to sit between the two electrons leads to an increased bonding between the electrons. This behavior is consistent with the fact that the binding energy of the charged exciton increases when a magnetic field is applied.

In Fig. $\Phi(a, b, c)$ we plot $|\Phi(x, 0,0)|^{2}$ for a charged exciton in a $100 \AA$ wide quantum well when the hole and one electron are fixed at a distance equal to their average position $d_{e h}=<\rho_{e h}^{2}>^{1 / 2}$, for the $B=0 \mathrm{~T}$ case (Fig. $\mathbb{1}(\mathrm{a})$ ), the $B=13.7 \mathrm{~T}$ case (Fig. $1(\mathrm{~b})$ ) and the $B=54 \mathrm{~T}$ case (Fig. $1(\mathrm{c})$ ). The qualitative difference, between the situation when a large magnetic field is applied and when a low magnetic field is applied is not very pronounced, except for the length scale. However, we observe that for $B=0 \mathrm{~T}$ the probability of having the second electron near the fixed electron is zero, while in the case in which a magnetic field is applied there is a finite probability for the second electron to be at the position of the first electron. Since the charged exciton is in the singlet state, the spin function is asymmetric for an interchange of the two electrons and consequently there is no Pauli exclusion principle to forbid the two electrons to be at the same position in space. Only the electron-electron interaction will make the latter probability as small as possible. This result is consistent with the result obtained for the pair-correlation functions.

Next we consider the triplet state and limit ourselves to the magnetic field $B=13.7 \mathrm{~T}$. Notice, that the triplet state is not bound for small magnetic fields. We plot $|\Phi(x, 0,0)|^{2}$ for a charged exciton in a $100 \AA$ wide quantum well when the two electrons are fixed (Fig. 5(a)) and when one electron and the hole are fixed (Fig. E(b)). Notice that there is not much qualitative difference between the conditional probability function of the triplet state and of the singlet state (see Fig. 3(b)). Quantitatively there are two major differences: i) the average distance between the particles is substantially larger, and ii) the probability to find the second electron at the same spatial position as the first one (see Fig. 5) is zero, while this is not the case for the singlet state. The latter is consistent with the fact that in the triplet state the electronic part of the wave function is antisymmetric under an exchange of the two electrons, which is also consistent with the fact that the electron-electron pair-correlation function is zero at the origin.

\section{COMPARISON OF THE TRANSITION ENERGIES WITH EXPERIMENTS}

In comparing our theoretical results with the available experimental data we assume that the observed peaks in the PL spectra are associated with an exciton, in which the electron and the hole recombine with emission of light, or with a recombination of a negatively-charged exciton, which leaves behind an electron in the lowest Landau level. Consequently, the transition energies are defined as

$$
\begin{gathered}
E_{X}=E_{g}+E(X), \\
E_{X^{-}}=E_{g}+E\left(X^{-}\right)-E_{e}(W, B),
\end{gathered}
$$

where $E_{g}$ is the energy band gap and $E_{e}(W, B)$ is the energy of a free electron in a quantum well of width W and in a magnetic field of strength $B ; E(X)$ and $E\left(X^{-}\right)$are, respectively, the exciton and charged exciton total energy. 
We will also take into account the Zeeman splitting induced by the magnetic field, under the assumption that the transitions observed follow the energy diagram discussed in Ref. 10. We also assume that the electron gyromagnetic factor, $g_{e}$, and the hole gyromagnetic factor are the same for the exciton as well as for the charged exciton. The total Zeeman splitting of each transition can then be written, in agreement with the results presented in Ref. 22, as

$$
\Delta E_{z}=\left(g_{e}+g_{h}\right) \mu_{B} B,
$$

where $\mu_{B}$ is the Bohr magneton. Notice also that the gyromagnetic factor is defined using the same conventions as in Ref. 22, i.e. the hole is considered to have an effective spin of $\Sigma_{h}=1 / 2$ instead of the real hole spin $S_{h}=3 / 2$. As a consequence of this Zeeman effect each transition line $E$ is split into two lines, i.e. $E^{ \pm}=E \pm \Delta E_{z} / 2$, associated to a change of 1 and -1 in the $z$-projection of the total angular momentum $\vec{J}=\vec{L}+\vec{S}$, i.e. $J_{z}$, respectively.

In Fig. 6 we compare our theoretical results for the transition energies of a $X^{-}$in a $300 \AA$ wide quantum well

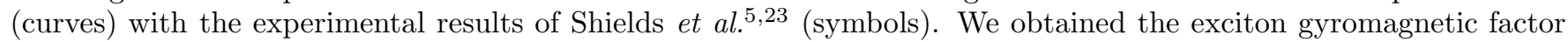
$g_{e x}=g_{e}+g_{h}=1.16$ from the measured splitting between the negatively $\left(\sigma_{-}\right)$and the positively (q- $)$circularly polarized lines of Ref. 5 using Eq. (8). This value of $g_{e x}$ is consistent with the results by Ossau et al.24 who found $g_{e x}=0.8$ for a $250 \AA$ wide quantum well. The experimental data presented in Fig. 6 are from the emitted negatively $\left(\sigma^{-}\right)$circular polarized light which results from transitions with $\Delta J_{z}=-1$. We choose the energy gap such that the exciton peak at $B=0 \mathrm{~T}$ coincides with the experimental exciton peak for $\mathrm{B}=0$, which leads to $E_{g}=1521.55$ $\mathrm{meV}$. Notice that for the singlet we reproduce the experimental behaviour, including the small minimum observed at low magnetic fields. Both for the exciton and for the triplet state of the charged exciton we find good agreement up to $8 \mathrm{~T}$. At small magnetic fields: 1) the theoretical results slightly overestimate the singlet transition energy which is probably a consequence of the importance of localization as argued, e.g., in Ref. 19, and 2) the tripletstate is unbound for small magnetic fields and consequently not observable. Notice also that the recently discussed 17 bright triplet (dotted curve) is not bound in the considered magnetic field region. None of the observed transitions can be associated to such a bright triplet. The data in the range 8-20 T are from Ref. 23 and are obtained under different experimental conditions as compared to those from Ref. 5 which were measured in the range 0-8 T. For example, an increase in electron density will shift the experimental photoluminescence towards larger energies. . $^{5}$ If we perform an uniform shift of the experimental data by $0.5 \mathrm{meV}$ in the 8-20 $\mathrm{T}$ range, which leads to the open symbols, a much better agreement with our theoretical results is obtained.

In Fig. $\mathrm{Z}(\mathrm{a}, \mathrm{b})$ we compare our theoretical results for the transition energies of a $100 \AA$ wide GaAs/AlGaAs quantum well with the experimental data obtained by Vanhoucke et al. 10 In Ref. 10 the Zeeman splitting was measured to be $\Delta E_{z} / B=0.11 \mathrm{meV} / \mathrm{T}$ leading to $g_{e x}=1.85$ which is very different from the value $g_{e x}=0.1$ obtained in Ref. 22 for a $115 \AA$ wide quantum well. The energy gap is fixed by matching the $B=5 \mathrm{~T}$ experimental and theoretical $X^{-}$singlet transition energies which resulted into $E_{g}=1520.35 \mathrm{meV}$. We use for the electron and the hole mass $m_{e}=0.067 m_{0}$ and $m_{h}=0.34 m_{0}$, respectively. The lower transition line (squares) is in rather good agreement with our results for the charged exciton singlet transition energy. For $B<3 \mathrm{~T}$ (see Fig. $\mathbb{7}(\mathrm{a})$ ) there is a substantial deviation between theory and experiment which again may be attributed to an enhancement of the negatively charged exciton binding energy due to localization of the trion. The higher transition line (circles) were attributed by the authors of Ref. 10 to the triplet charged exciton. Our theoretical results agree with this assignment at least for $B<20 \mathrm{~T}$ (Fig. 7(a)). Notice that this magnetic field range, i.e. $B<18 \mathrm{~T}$, is the same studied in Fig. 6 for the $300 \AA$ quantum well. In the high magnetic field range (Fig. $\mathrm{G}(\mathrm{b})$ ), i.e. $B>25 \mathrm{~T}$, the experimental results follow very closely the theoretical exciton transition energy, which coincides practically with the $X^{-}$bright triplet transition energy. In the intermediate magnetic field range, i.e. $18 \mathrm{~T}<B<25 \mathrm{~T}$ the results transit from the $X^{-}$triplet to the exciton transition or bright triplet transition.

From the above comparison we may construct the following picture: 1) in the magnetic field range $B<18 \mathrm{~T}$ quantum well width fluctuations and disorder break the translational invariance of the system which results into a breakdown of the optical selection rule, thus allowing the dark triplet negatively charged exciton state to be optically active; 2 ) only in the very small magnetic field range, i.e. $B<5 \mathrm{~T}$, the localization of the trion due to quantum well width fluctuations leads to an increase of the singlet and triplet $X^{-}$binding energy. For the $300 \AA$ wide quantum well the effect of the quantum well width fluctuations on the trion energy is substantially smaller. 19 This agrees with Fig. 6 where the magnetic field range over which the singlet binding energy is strongly enhanced is much smaller, i.e. $B<2 \mathrm{~T}$, and the size of the enhancement is also substantially smaller; 3 ) in the very large magnetic field range $B>25 \mathrm{~T}$ the optical selection rule is restored and no transition from the $X^{-}$dark triplet is observed. Because of the inhibition of the decay of the $X^{-}$dark triplet it is possible that the bright triplet becomes sufficiently populated making it experimentally observable. We found that this $X^{-}$bright triplet is at most marginally bound and therefore has almost the same transition energy as the exciton.

For $B>40 \mathrm{~T}$ the experimental results are slightly lower in energy as compared to our theoretical results. A possible reason for this deviation may be the importance of band non-parabolicity at such large magnetic fields. For example, 
if we increase the hole mass to $m_{h}=0.37 m_{0}$ at $B=50 \mathrm{~T}$, the $X^{-}$singlet (exciton) transition energy becomes 1.5780 $\mathrm{eV}(1.5812 \mathrm{eV})$ which is almost $2 \mathrm{meV}$ lower than the $m_{h}=0.34 m_{0}$ result $1.5796 \mathrm{eV}(1.5824 \mathrm{eV})$, thus proving a strong dependence of the transition energy on the hole mass value. This is mainly due to the difference in confinement energy. Notice, that the binding energy only changes from $2.8 \pm 0.1 \mathrm{meV}$ to $3.2 \pm 0.1 \mathrm{meV}$, showing a less strong dependence on the hole mass.

\section{COMPARISON OF THE TRION BINDING ENERGY WITH EXPERIMENTS AND WITH OTHER THEORETICAL RESULTS}

Finally we compute the binding energy of the negatively charged exciton and compare it with the available experimental results. The binding energy is defined as

$$
E_{B}\left(X^{-}, B\right)=E(X)+E_{e}(W, B)-E\left(X^{-}\right),
$$

where $E(X)$ and $E\left(X^{-}\right)$are respectively, the total energy of an exciton and of a charged exciton in the quantum well and $E_{e}(W, B)$ is the energy of a single electron in the quantum well of width W.

In Fig. 8 we present our results for the binding energy of a negatively charged exciton in a $300 \AA$ wide GaAs $/ \mathrm{Al}_{0.3} \mathrm{Ga}_{0.7} \mathrm{As}$ quantum well and we compare it with the experimental binding energy obtained by Shields et al. 23 (symbols) and with the theory of Whittaker and Shield 12 (dotted and dash-dotted curves). The error bars in the figure indicate the estimated accuracy of our results. Note that the electron spin-singlet binding energy (solid curve) increases with magnetic field, up to about $35 \mathrm{~T}$, after which it saturates. The electron spin-triplet binding energy (dashed curves) smoothly increases with magnetic field up to $60 \mathrm{~T}$. Notice the very good agreement between our theory and the experimental binding energies both for the singlet and triplet state up to about 13 T. For the lower magnetic field range, $B<2 \mathrm{~T}$, the binding energies are slightly underestimated theoretically. We believe that the larger binding energy obtained experimentally is a consequence of the localization of the trion, as already noticed for the $B=0 \mathrm{~T}$ case.19 The effect of the magnetic field, however, decreases the discrepancy between theory and experiment. This is due to the fact that the magnetic field increases the localization of the charged exciton, which is then less sensitive to the well width fluctuations. In the range $8 \mathrm{~T} \leq B \leq 20 \mathrm{~T}$ the experimental binding energies show almost no magnetic field dependence which is in contrast to our theoretical results which still increases with $B$, although less fast than for $B \leq 8 \mathrm{~T}$. As already mentioned the $8 \mathrm{~T} \leq B \leq 20 \mathrm{~T}$ experimental results are measured under different experimental conditions than those in the region $B \leq 8 \mathrm{~T}$. Notice that our singlet binding energy is considerably larger than the one obtained by Whittaker and Shields, 12 while the triplet binding energy is comparable to the one of Ref. 12 up to $15 \mathrm{~T}$. For $B>15 \mathrm{~T}$ the present triplet binding energy becomes appreciably larger than the one of Ref. 12. One of the reasons for this differences between our results and those of Whittaker and Shields are the different parameters used in Ref. 12. They used $m_{h \|}^{w}=0.34 m_{0}, m_{e}^{w}=0.065 m_{0}$ in the well, $m_{h \|}^{b}=0.45 m_{0}$, $m_{e}^{b}=0.07 m_{0}$ in the barrier and $m_{h \perp}=0.18 m_{0}$ in the well and in the barrier, which partially explains the lower binding energy.

The binding energy for a charged exciton in a $100 \AA$ wide $\mathrm{GaAs} / \mathrm{Al}_{0.3} \mathrm{Ga}_{0.7}$ As quantum well is shown in Fig. 9 and compared to the theory of Whittaker and Shields 12 (dotted and dash-dotted curves). Notice that: i) we find substantial larger binding energies than Whittaker and Shields, 12 ii) no crossing between the singlet and the triplet energies is found at least up to $70 \mathrm{~T}$, while Whittaker and Shields predicted a singlet-triplet crossing near $30 \mathrm{~T}$, and iii) the bright triplet is at most marginally bound for $B>5 \mathrm{~T}$. We find a binding energy of $0.15 \pm 0.1 \mathrm{meV}$ while Wojs et al. 17 obtained a binding energy of $0.75 \mathrm{meV}$ for $B=20 \mathrm{~T}$ (in Ref. 26 a reduced binding energy of $0.37 \mathrm{meV}$ was reported). For the $300 \AA$ wide quantum well we found that the bright triplet state was unbound for the considered magnetic field range.

The quantitative discrepancy between our theoretical results and the one of Ref. 17 is probably a consequence of the approximations made by the authors of Ref. 17: i) they replace the real quantum well $W$ with a hard wall quantum well with an effective width and only the lowest subband is retained, ii) the $3 \mathrm{D}$ problem is replaced by an effective $2 \mathrm{D}$ problem (in which the Coulomb interaction is approximated by the $2 \mathrm{D}$ screened interaction: $e^{2} / \epsilon \sqrt{\rho^{2}+\lambda^{2}}$ ), iii) the flat 2D quantum well geometry is replaced by a Haldane sphere and iv) only the lowest 5 single particle Landau levels are included in their wave function. Previously we showed $1 \mathrm{~g}$ for $\beta=0$, that the approximations i) and ii) lead to an overestimation of the binding energy of the charged exciton 19 Whittaker and Shields 12 showed that the inclusion of higher subbands and of higher Landau Levels in the wave function substantially increases the high field singlet binding energy, while they have a smaller effecton the triplet binding energy.

Note that in agreement with Whittaker and Shields, 12 and in contrast to the recent work by Stébé and Moradi, 13 we find that the spin-triplet state is unbound for $B=0 \mathrm{~T}$. This disagreement with the work of Stébé and Moradil 3 
can be traced back to their poor variational function which gives an exciton energy which is about $8 \%$ larger than ours, while the negatively charged exciton singlet energy is about $5 \%$ lower than ours.

It has been argued that the hole mass is asymmetric and that the in-plane hole mass depends on the magnetic field. One expects that the hole mass in the z-direction, i.e. the confinement direction, will almost not influence the exciton and trion binding energies. This is different for the in-plane hole mass which, e.g. through the reduced exciton mass $\mu$, will change the exciton and to a lesser extent the trion energies. In a recent cyclotron resonance experiment by Cole et al.27 on p-doped (311)A GaAs quantum wells the measured hole mass varies from $m_{h} \approx 0.15-0.18 m_{0}$ for $B<5 \mathrm{~T}$ to $m_{h} \approx 0.35 m_{0}$ at higher fields for a $150 \AA$ wide quantum well. For wider wells the large hole mass value was reached at smaller magnetic fields and therefore, this mass variation is expected not to be relevant for the 300 A sample. In order to investigate the influence of the value of the in-plane hole mass on the trion singlet and triplet binding energy we compare in Fig. 9 our results with those for the asymmetric hole mass (thin solid and dashed curves in Fig. 9) in which the in-plane hole mass was reduced to $m_{h}=0.18 m_{e}$. Notice that: i) the singlet trion binding energy is substantially reduced (about $0.5 \mathrm{meV}$ ); ii) the triplet binding energy is practically not altered and coincides with the Whittaker and Shields 12 results for $B<15 \mathrm{~T}$, and iii) there is a singlet-triplet crossing at about $40 \mathrm{~T}$. With this smaller hole mass the exciton reduced mass is diminished by $13 \%$ leading to a lower exciton binding energy and also to an increase of the trion total energies. This shifts the theoretical curves in Fig. 17 in such a way that an unrealistic low band gap of $1518.3 \mathrm{meV}$ has to be assumed in order to match the experimental and theoretical $B \approx 5 \mathrm{~T}$ trion transition energies. Furthermore, the agreement between theory and experiment is lost for $B>10 \mathrm{~T}$ and the experimental trion singlet energy for $B<3 \mathrm{~T}$ is now higher than the theoretical curve which disagrees with the idea of an enhanced trion binding energy in this low field region due to quantum well width fluctuations. These findings argue against such a reduced hole mass, even in the low magnetic field range.

It should also be noted that the use of a cyclotron mass in our calculation may be questionable. In a cyclotron resonance experiment, transitions between two Landau Levels are induced and from the transition energy $\hbar \omega^{*}=$ $E_{1}-E_{0}$ one defines the cyclotron mass $m_{c}^{*}=e B / c \omega^{*}$, where $E_{n}$ is the energy of the n-th Landau Level. Notice that such a definition only corresponds to the effective hole mass if the hole mass is independent of the Landau Level. Furthermore, e.g., electric subband crossings and polaron effects may invalidate such an assignment. A further argument against the use of the low magnetic field cyclotron hole mass published by Cole et al. 27 is that those results are for the (311) GaAs plane while the experiments of Vanhoucke et al.10 were performed on samples with quantum wells in the (100) plane. It is well known that in the latter crystallographic direction, with increasing density or increasing magnetic field, the hole mass very quickly reaches a value in the $m_{h} \approx 0.3-0.5 m_{0}$ range, the exact value depends on the quantum well width (see for example Ref. 28). We believe that this argues in favor of the use of $m_{h}=0.34 m_{0}$ in the important $B>4 \mathrm{~T}$ magnetic field region as we did.

For the $100 \AA$ wide quantum well no experimental results on the trion binding energy are available. Therefore, we show in Fig. 10 the energy difference between the two transition lines as measured in Ref. 10 and compare them with: 1) the negatively charged exciton singlet binding energy (solid curve), 2) the energy difference between the negatively charged exciton dark triplet and singlet (dashed curve), 3) the energy difference between the negatively charged exciton bright triplet and singlet (dotted curve). To be complete we also show the negatively charged exciton bright triplet binding energy. This figure nicely illustrates how in the low magnetic field region, and more precisely in the range 6-18 $\mathrm{T}$ the experimental results are clearly not related to the binding energy of the $X^{-}$singlet state but rather to the difference between the dark triplet state and the singlet state energy. In the high magnetic field region, i.e. $B>25 \mathrm{~T}$, the experimental results are closer to the singlet state binding energy and to the energy difference between the bright triplet state and the singlet state.

\section{CONCLUSION}

We presented a calculation of the lowest energy levels of the negatively charged exciton spectrum in a quantum well and in the presence of a magnetic field which is perpendicular to the quantum well plane. Our approach is based on the stochastic variational method in which the trion wavefunction is expanded in deformed correlated Gaussian functions. The important correlation between the particles is built in this wavefunction and therefore such an approach is well suited for problems in which the binding of the system is a pure consequence of the particle-particle correlation as is the case for the trion. We do not observe any spin-singlet/spin-triplet transition using the symmetric mass approximation, however such a transition is found for the $100 \AA$ wide quantum well if we use the asymmetric hole mass approximation (i.e. a substantially lower in-plane hole mass), in agreement with what was predicted by Whittaker and Shields. 12 The singlet-triplet transition is found to occur at about $40 \mathrm{~T}$, in contrast to the predicted $B=30 \mathrm{~T}$ reported in Ref. 12. We have argued that at such high magnetic fields the larger in-plane hole mass should be used and consequently we believe that this transition should not occur in reality for $B<70 \mathrm{~T}$. Muntenau et al 14 observed a spin-singlet/spin-triplet 
transition in an asymmetric quantum well in which electrons and holes are spatially separated. Such a sipglet-triplet transition is then of the same nature as the one predicted for spatially separated charged donor systems. 26.69 .30

A comparison between our theoretical results and available experiments gives good agreement for the trion singlet and triplet energy. Particular good agreement is achieved with the experimental results of Shields et al Ele $23_{\text {on }}$ the $300 \AA$ quantum well. For the results on the $100 \AA$ quantum well we find good agreement for the trion singlet state while for the higher energy transition we find for $B<20 \mathrm{~T}$ that the results agree with the dark triplet transition, while for $B>25 \mathrm{~T}$ this transitions agrees more closely with the exciton transition energy or the bright triplet energy. Because the latter two have, in this magnetic field region, practically the same energy we are not able to make any definite assignment for this transition line.

\section{ACKNOWLEDGMENT}

Part of this work is supported by the Flemish Science Foundation (FWO-Vl), the 'Interuniversity Poles of Attraction Program - Belgian State, Prime Minister's Office - Federal Office for Scientific, Technical and Cultural Affairs', the "Onderzoeksraad van de Universiteit Antwerpen", and the Flemish-Hungarian Cultural exchange program. K. Varga was supported by the U. S. Department of Energy, Nuclear Physics Division, under contract No. W-31-109-ENG39 and OTKA grant No. T029003 (Hungary). Discussions with M. Hayne, T. Vanhoucke, A. Dzyubenko and correspondence with A. Wojs are gratefully acknowledged.

* Electronic address: riva@uia.ua.ac.be.

- Electronic address: peeters@uia.ua.ac.be.

¥ On leave of absence from: Institute of Nuclear Physics of the Hungarian Academy of Sciences, Debrecen, Hungary.

${ }^{1}$ M. A. Lampert, Phys. Rev. Lett. 1, 450 (1958).

${ }^{2}$ G. Munschy and B. Stébé, Phys. Stat. Sol. (b) 64, 213 (1974).

${ }^{3}$ B. Stébé and A. Aniane, Superlatt. Microstruct. 5, 545 (1989).

${ }^{4}$ K. Kheng, R. T. Cox, Y. Merle d'Aubigne, F. Bassani, K. Saminadayar, and S. Tatarenko, Phys. Rev. Lett. 71, 1752 (1993); K. Kheng, K. Saminadayar, and N. Magnea, J. Cryst. Growth 184/185, 849 (1998).

${ }^{5}$ A. J. Shields, M. Pepper, M. J. Simmons, and D. A. Ritchie, Phys. Rev. B 52, 7841 (1995).

${ }^{6}$ G. Finkelstein, H. Shtrikman, and I. Bar-Joseph, Phys. Rev. B 53, R1709 (1996); G. Finkelstein, H. Shtrikman, and I. Bar-Joseph, Phys. Rev. B 53, 12593 (1996).

${ }^{7}$ S. Glasberg, G. Finkelstein, H. Strikman, and I. Bar-Joseph, Phys. Rev. B 59, R10425 (1999).

${ }^{8}$ M. Hayne, C. L. Jones, R. Bogaerts, C. Riva, A. Usher, F. M. Peeters, F. Herlach, V.V. Moshchalkov, and M. Henini, Phys. Rev. B 59, 2927 (1999).

${ }^{9}$ Y. Kim, F. M. Muntenau, C. H. Perry, D. G. Rickel, J. A. Simmons, and J. L. Reno, Phys. Rev. B 61, 4492 (2000).

10 T. Vanhoucke, M. Hayne, V. V. Moshchalkov, and M. Henini, Solid Stat. Commun. 115, 403 (2000).

11 A. J. Shields, J. L. Osborne, D. M. Whittaker, M. Y. Simmons, M. Pepper, and D. A. Ritchie, Phys. Rev. B 55, 1318 (1997); A. J. Shields, F. M. Bolton, M. Simmons, M. Pepper, and D. A. Ritchie, Phys. Rev. B 55, R1970 (1997).

${ }^{12}$ D. M. Whittaker and A. J. Shields, Phys. Rev. B 56, 15185 (1997).

13 B. Stébé and A. Moradi, Phys. Rev. B 61, 2888 (2000).

${ }^{14}$ F. M. Muntenau, Y. Kim, C. H. Perry, D. G. Rickel, J. A. Simmons, and J. L. Reno, Phys. Rev. B 61, 4731 (2000).

15 A. B. Dzyubenko and A. Yu. Sivanchenko, Physica E 6, 226 (2000);

${ }^{16}$ A. B Dzyubenko, Solid Stat. Commun. 113, 683 (2000).

17 A. Wojs, J. J. Quinn, and P. Hawrylak, Phys. Rev. B 62, 4630 (2000).

${ }^{18}$ C. Riva, F. M. Peeters, and K. Varga, Phys. Stat. Sol.(a) 178, 513 (2000).

${ }^{19}$ C. Riva, K. Varga, and F. M. Peeters, Phys. Rev. B. 61, 13873 (2000).

${ }^{20}$ B. Stébé, E. Feddi, A. Ainane, and F. Dujardin, Phys. Rev. B 58, 9926 (1998).

${ }^{21}$ Y. Suzuki and K. Varga, Stochastic Variational Approach to Quantum-Mechanical Few-Body Problems (Springer, BerlinHeidelberg, 1998).

${ }^{22}$ M. J. Snelling, G. P. Flinn, A. S. Plaut, R. T. Harley, A. C. Tropper, R. Eccleston, and C. C. Phillips, Phys. Rev. B 44, 11345 (1991); M. J. Snelling, E. Blackwood, C. J. McDonagh, R. T. Harley, and C. T. B. Foxon, Phys. Rev B 45, 3922 (1992).

${ }^{23}$ A. J. Shields, M. Pepper, P. C. M. Christiansen, J. C. Maan, M. Y. Simmons, and D. A. Ritchie, Proc. 12th International 
Conference on High Magnetic Fields in Physics of Semiconductors II, Vol. 2, Eds. G. Landwehr and W. Ossau (World Scientific, Singapore, 1997) p. 737.

${ }^{24}$ W. Ossau, B. Jäkel, E. Bangert, And G. Weimann, NATO Advanced Study Institutes Ser. B, Vol. 183, Eds. C. Y. Fong, I. P. Batra, and C. Cirac (Plenum, New York, 1988), p. 285.

${ }^{25}$ V. Huard, R. T. Cox, K. Saminadayar, A. Arnoult, and S. Tatarenko, Phys. Rev. Lett. 84, 187 (2000).

${ }^{26}$ I. Szlufarska, A. Wójs, and J. J. Quinn, cond-mat/0009251.

${ }^{27}$ B. E. Cole, J. M. Chamberlain, M. Henini, T. Cheng, W. Batty, A. Wittlin, J. A. A. J. Perenboom, A. Ardavan, A. Polisski, and J. Singleton, Phys. Rev. B 55, 2503 (1997).

${ }^{28}$ G. Goldoni and F. M. Peeters, Phys. Rev. B 51, 17806 (1995).

${ }^{29}$ I. K. Marmorkos, V. A. Schweigert, and F. M. Peeters, Phys. Rev. B 55, 5065 (1997).

${ }^{30}$ C. Riva, V. A. Schweigert, and F. M. Peeters, Phys. Rev. B 57, 15392 (1998)

FIG. 1. The 2D average interparticle distance vs. the magnetic field for the exciton, and the singlet and triplet state of the charged exciton in a quantum well of width $100 \AA$.

FIG. 2. The 2D pair correlation function vs. the magnetic field for the exciton and the spin-singlet and spin-triplet state of a charged exciton in a $100 \AA$ wide quantum well.

FIG. 3. The projection on the $\mathrm{x}$-axis of the conditional probability for the charged exciton for $B=0 \mathrm{~T}(\mathrm{a}), B=13.7 \mathrm{~T}(\mathrm{~b})$ and for $B=54 \mathrm{~T}$ (c) in a quantum well of width $100 \AA$. The symbols represent the fixed electrons.

FIG. 4. The projection along the x-axis of the conditional probability for the charged exciton, for $B=0 \mathrm{~T}$ (a), $B=13.7 \mathrm{~T}$ (b) and for $B=54 \mathrm{~T}$ (c) in a quantum well of width $100 \AA$. The symbols represent the fixed electron and the hole.

FIG. 5. The projection on the $\mathrm{x}$-axis of the conditional probability function for the triplet state, when the electrons are fixed (a) and when one electron and the hole are fixed (b), in quantum well of width $100 \AA$ and for $B=13.7$ T. The symbols represent the fixed particles.

FIG. 6. Comparison between the experimental and theoretical transition energies for charged excitons and excitons in a 300 $\AA$ wide quantum well. The open symbols are the experimental results for $B>8 \mathrm{~T}$ shifted by $0.5 \mathrm{meV}$.

FIG. 7. Comparison between the experimental and the theoretical transition energies for charged excitons and excitons in a $100 \AA$ wide quantum well. For clarity, the low magnetic field region (a) and the high magnetic field region (b) are shown separately.

FIf The binding energy of a charged exciton in a $300 \AA$ widequantum well compared to the experimental data of Shields et al. $\mathbf{H}_{23}$ and to the theoretical results by Whittaker and Shields. 12

FIG. 9. The binding energy of a charged exciton in a $100 \AA$ wide quantum well calculated using the symmetric hole mass approximation (thick curves) and the asymmetric hole mass approximation (thin curves). The results are compared to the theoretical results by Whittaker and Shields.12

FIG. 10. Comparison of the difference in energy between the upper and lower $\sigma^{-}$transition lines in Ref. 10 (symbols) with our theoretical binding energy for the negative trion singlet state (solid curve), the energy difference between our theoretical dark triplet and singlet state (dashed curve) and the energy difference between our bright triplet and singlet state (dotted curve). 


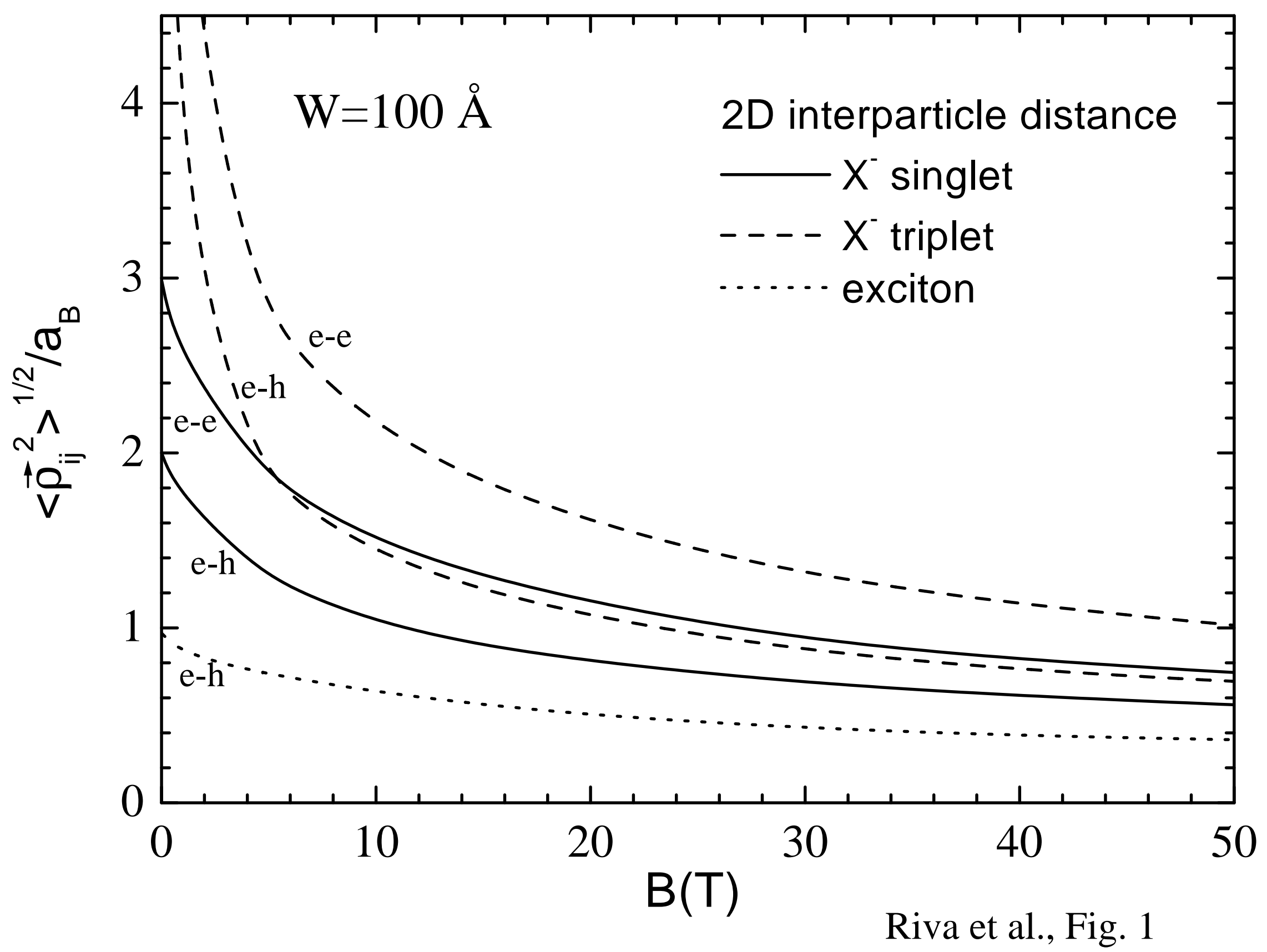




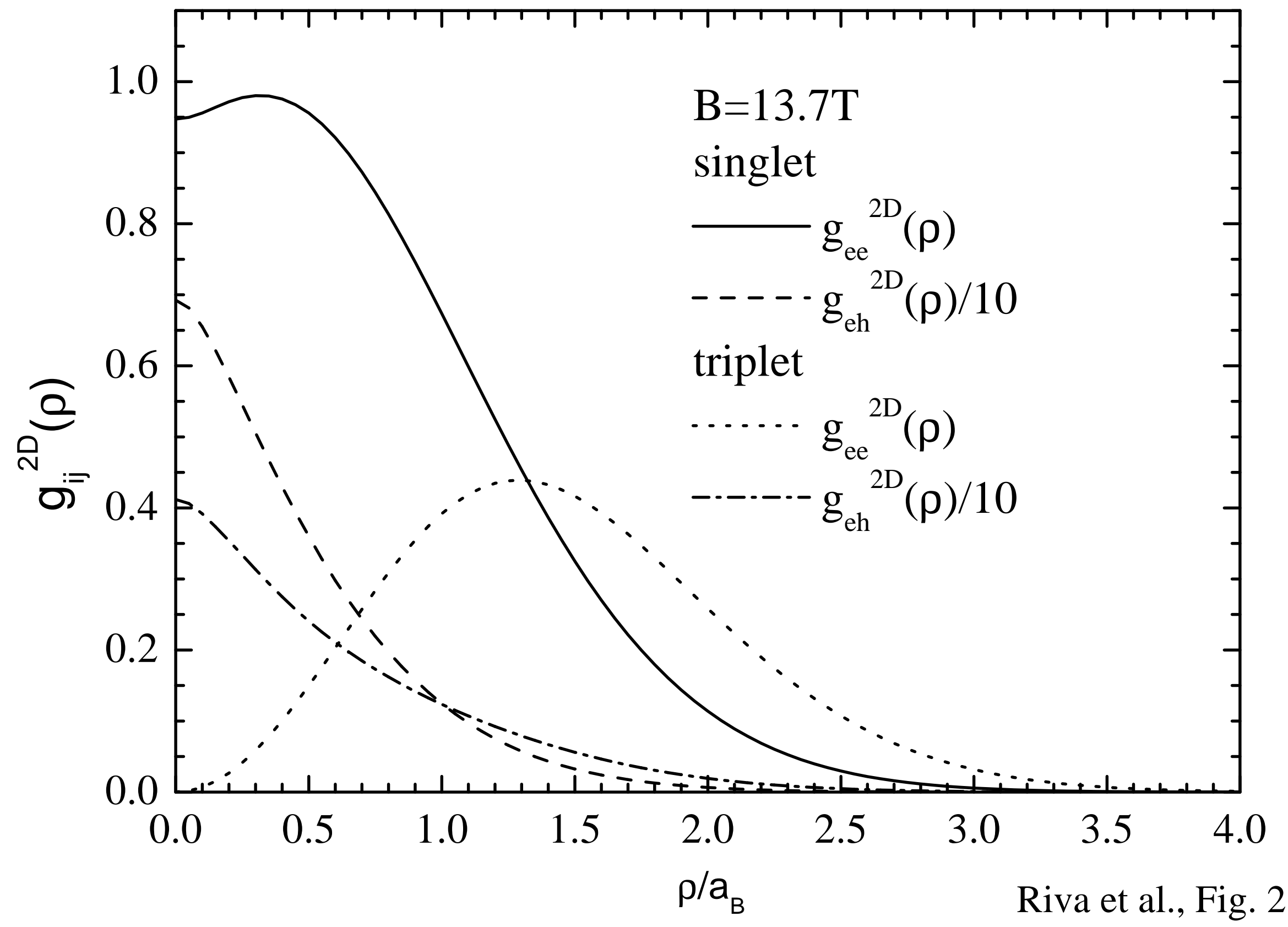




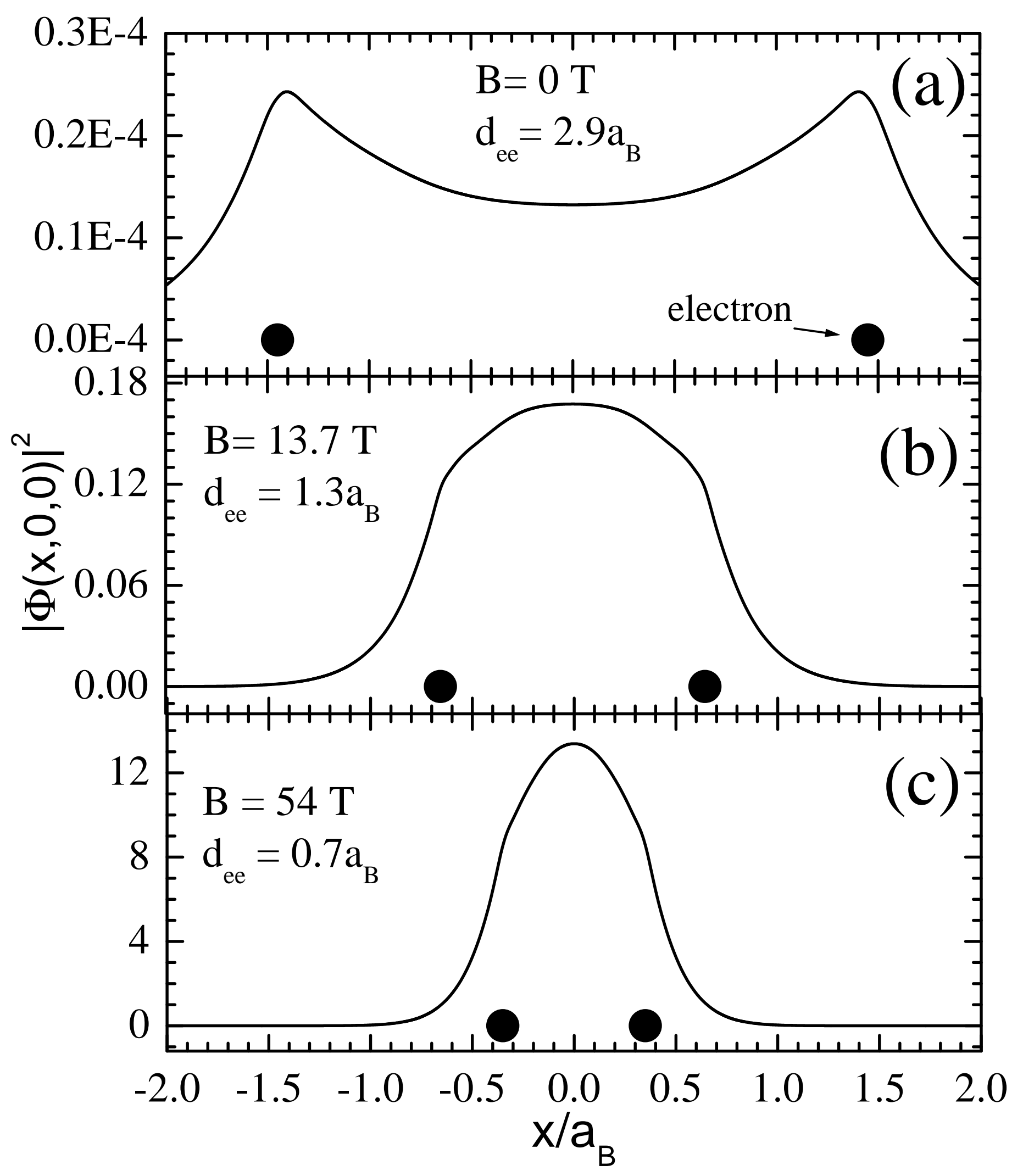

Riva et al., Fig. 3 


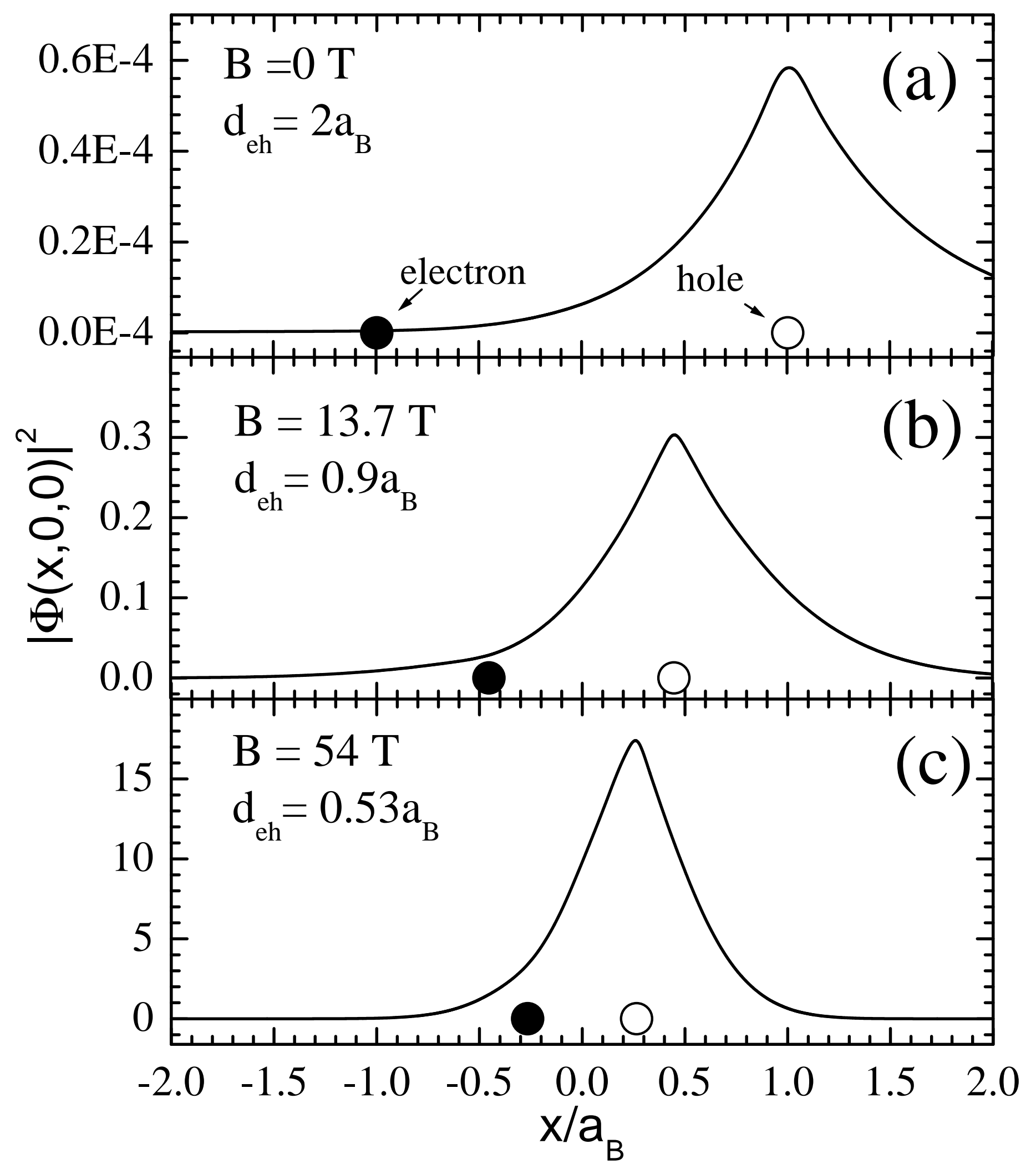

Riva et al., Fig. 4 


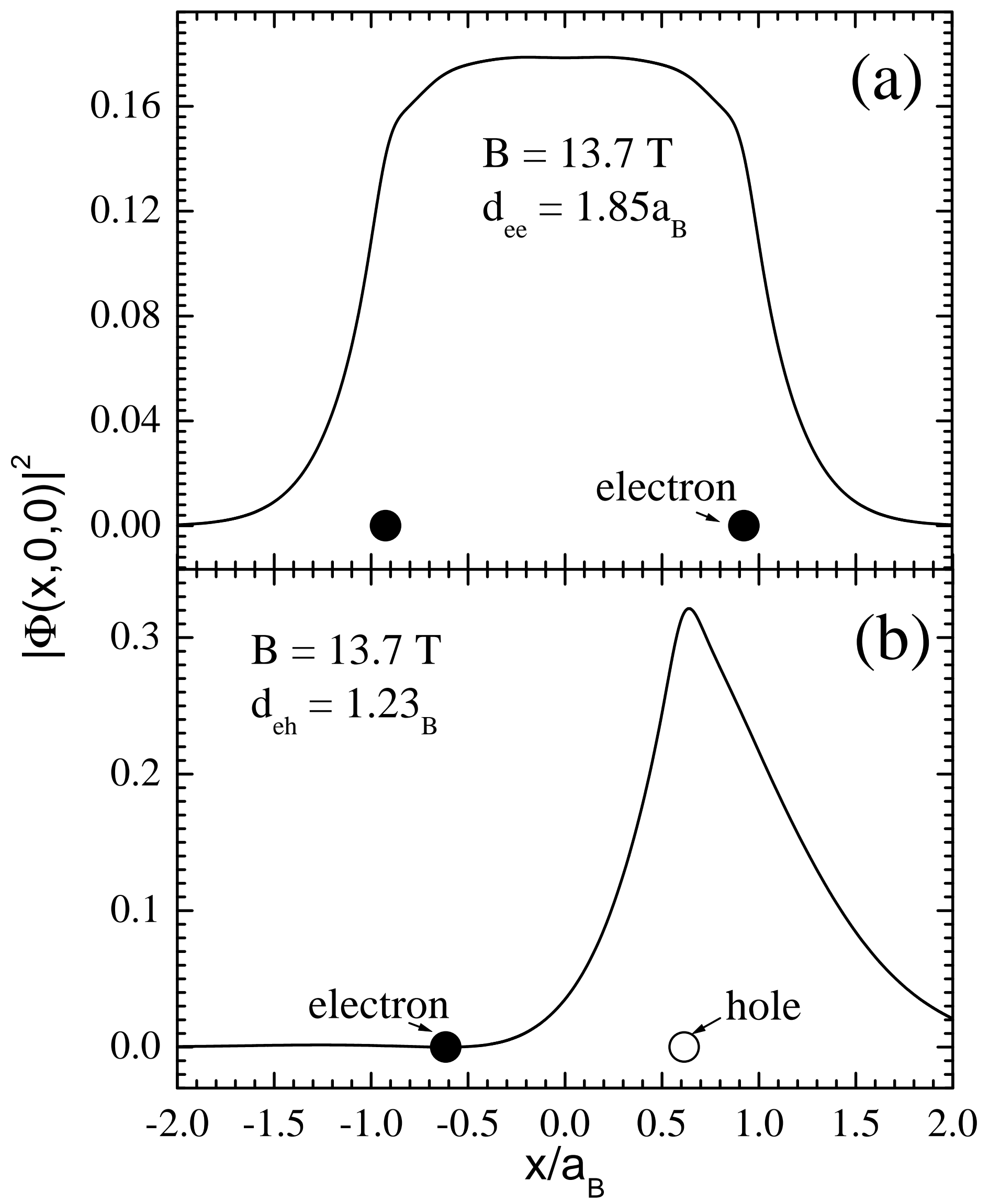

Riva et al., Fig. 5 


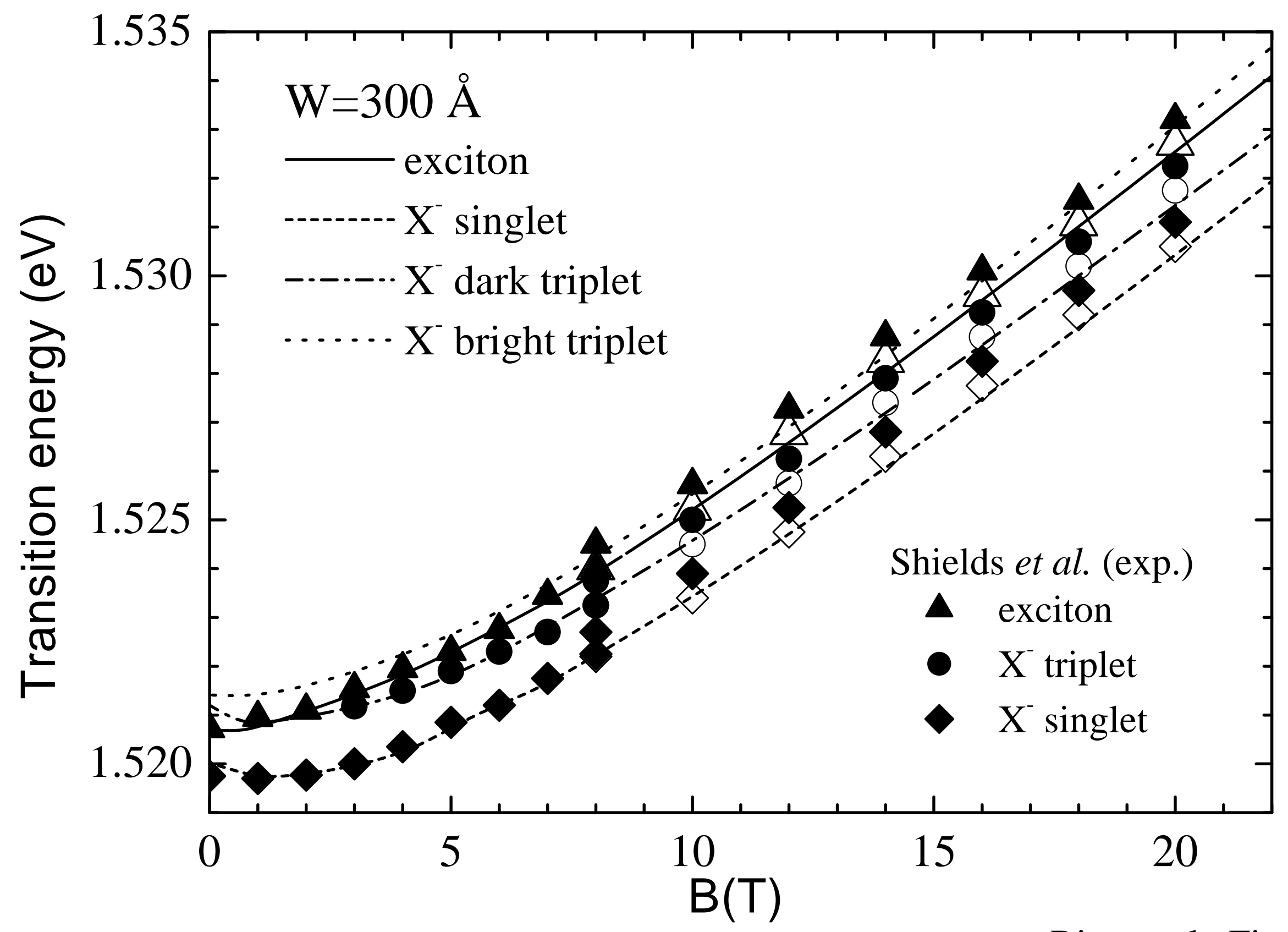

Riva et al., Fig. 6 

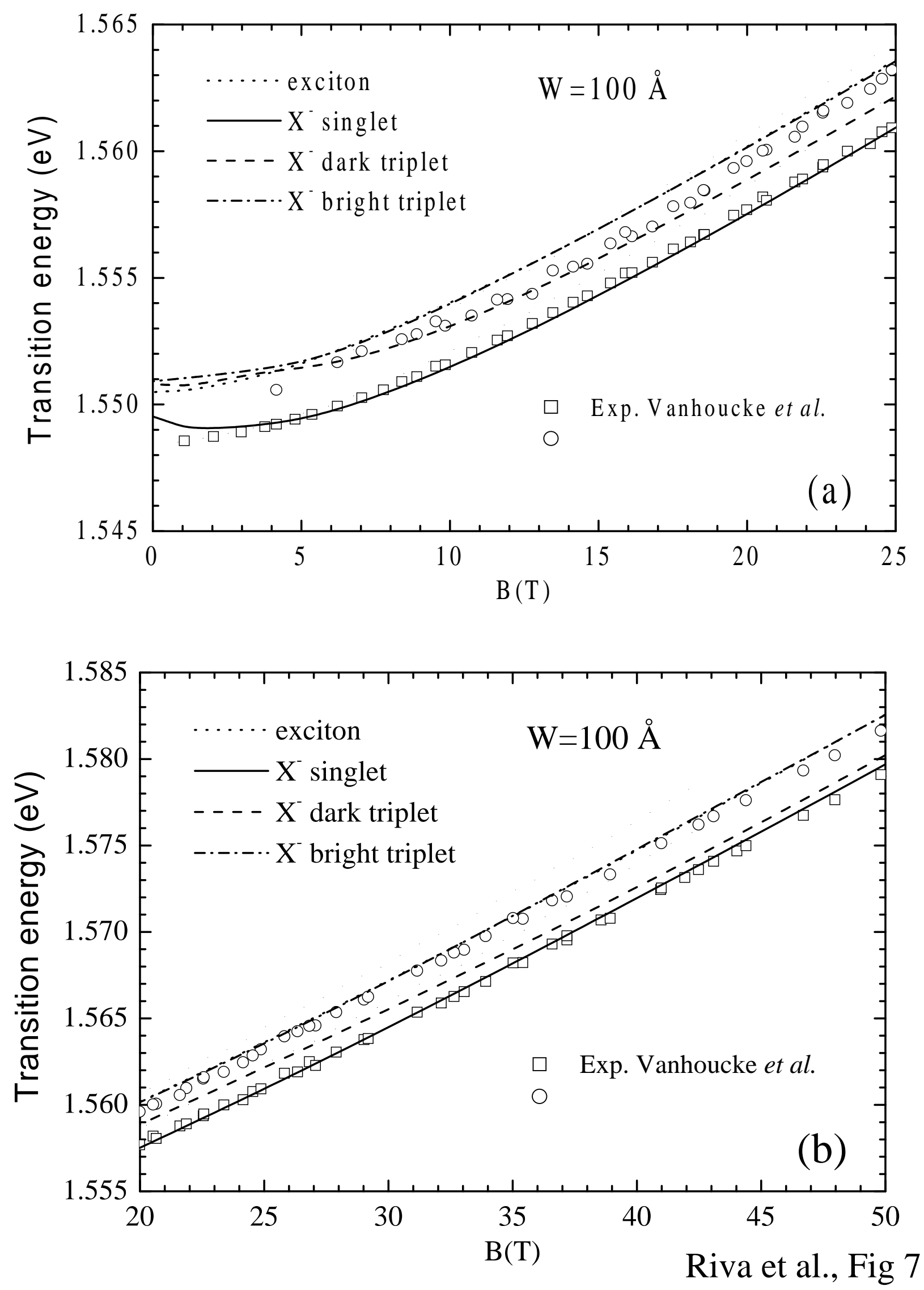


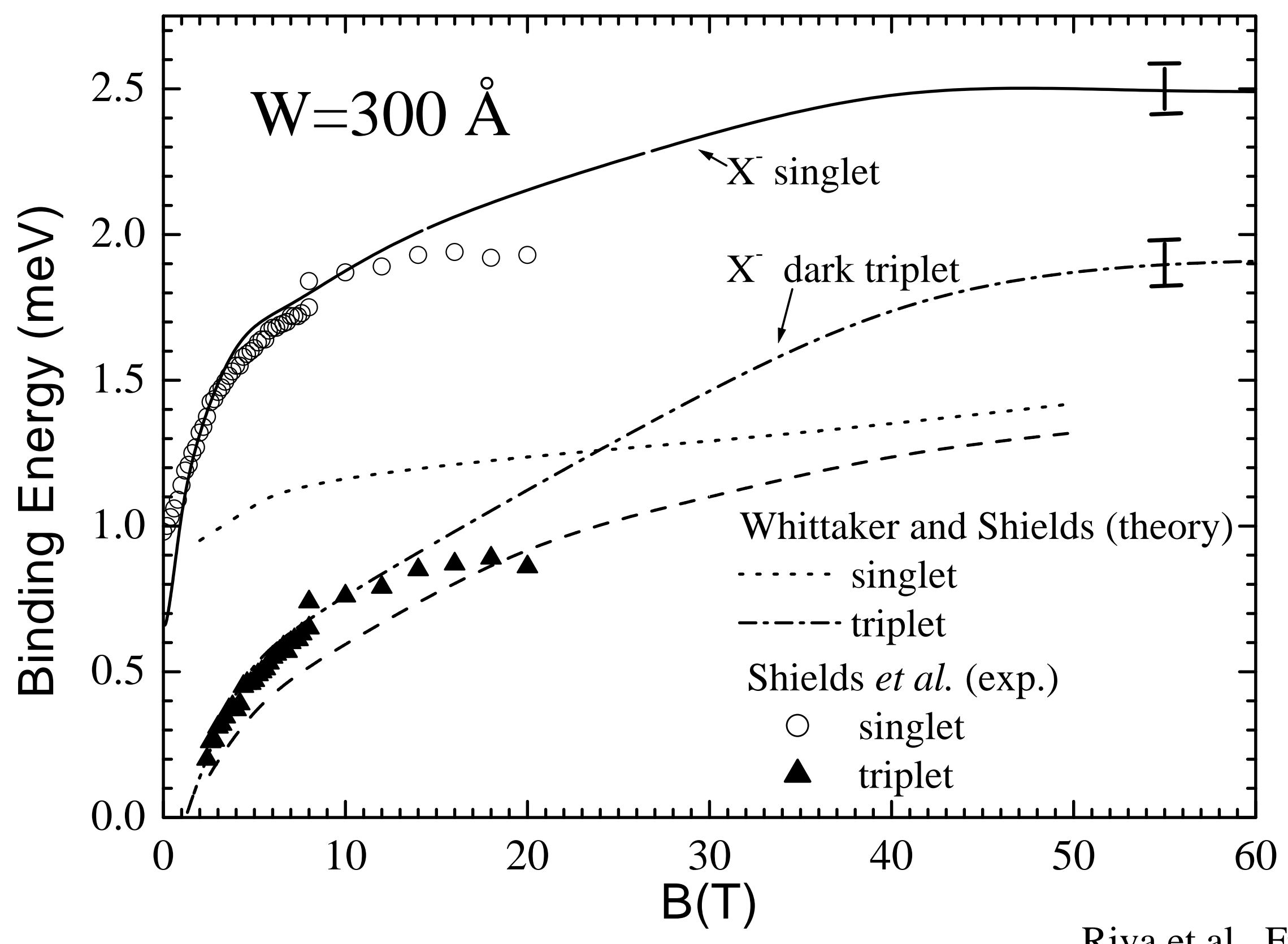

Riva et al., Fig. 8 


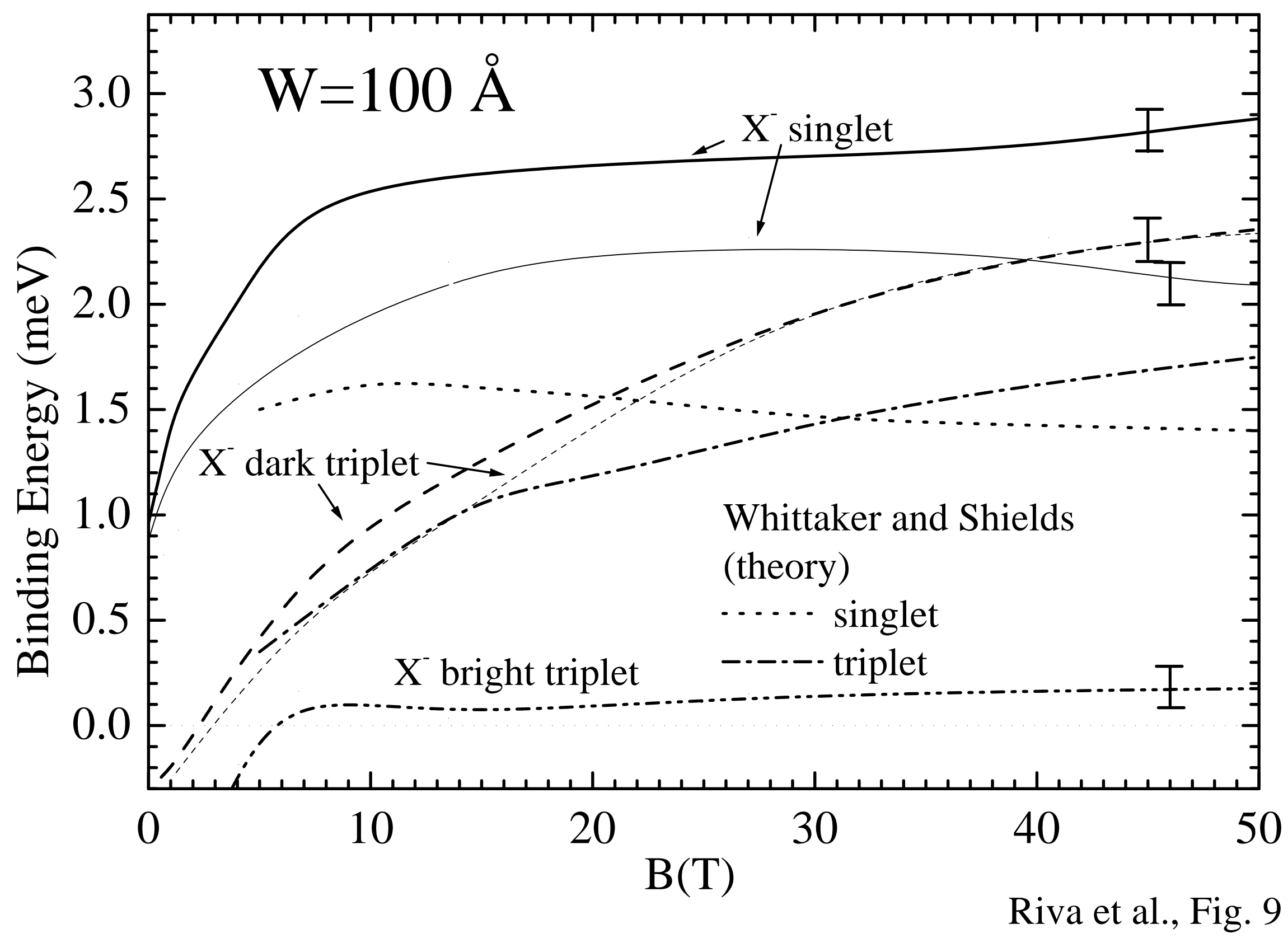




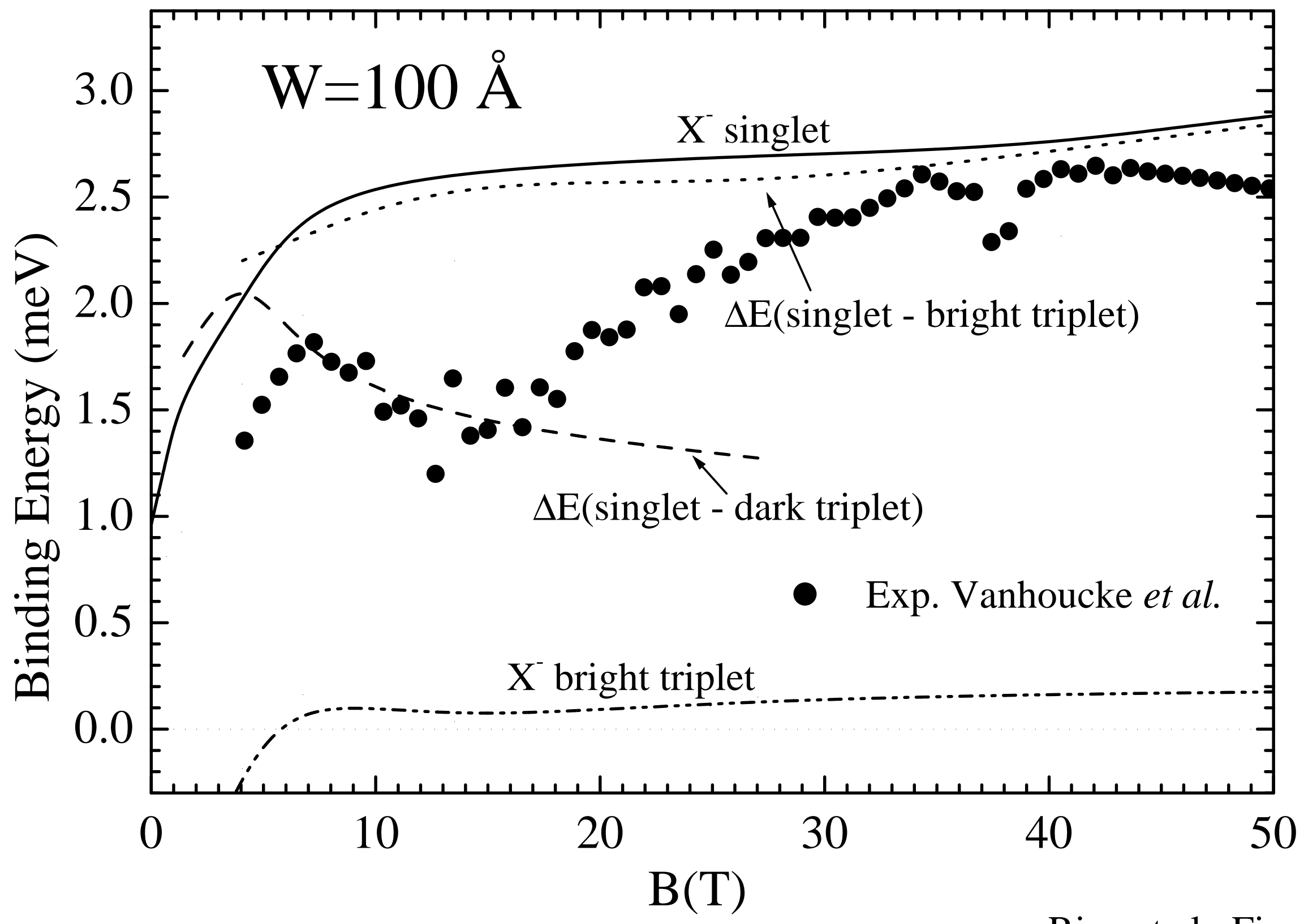

Riva et al., Fig. 10 